\title{
Correct Folding of the $\beta$-Barrel of the Human Membrane Protein VDAC Requires a Lipid Bilayer
}

\author{
Baladhandapani Shanmugavadivu ${ }^{1}$, Hans-Jürgen Apell ${ }^{1}$ \\ Thomas Meins $^{2}$, Kornelius Zeth ${ }^{2}$ and Jörg H. Kleinschmidt ${ }^{1 *}$
}

\author{
${ }^{1}$ Fachbereich Biologie \\ Universität Konstanz \\ Universitätsstraße 10 \\ D-78464 Konstanz \\ Germany \\ ${ }^{2}$ Max-Planck-Institut \\ für Biochemie \\ Abt. Membranbiochemie \\ Am Klopferspitz 18 \\ D-82152 Martinsried \\ Germany
}

\begin{abstract}
Spontaneous membrane insertion and folding of $\beta$-barrel membrane proteins from an unfolded state into lipid bilayers has been shown previously only for few outer membrane proteins of Gram-negative bacteria. Here we investigated membrane insertion and folding of a human membrane protein, the isoform 1 of the voltage-dependent anion-selective channel (hVDAC1) of mitochondrial outer membranes. Two classes of transmembrane proteins with either $\alpha$-helical or $\beta$-barrel membrane domains are known from the solved high-resolution structures. VDAC forms a transmembrane $\beta$-barrel with an additional $N$-terminal $\alpha$-helix. We demonstrate that similar to bacterial OmpA, urea-unfolded hVDAC1 spontaneously inserts and folds into lipid bilayers upon denaturant dilution in the absence of folding assistants or energy sources like ATP. Recordings of the voltage-dependence of the single channel conductance confirmed folding of hVDAC1 to its active form. hVDAC1 developed first $\beta$-sheet secondary structure in aqueous solution, while the $\alpha$-helical structure was formed in the presence of lipid or detergent. In stark contrast to bacterial $\beta$ barrel membrane proteins, hVDAC1 formed different structures in detergent micelles and phospholipid bilayers, with higher content of $\beta$-sheet and lower content of $\alpha$-helix when inserted and folded into lipid bilayers. Experiments with mixtures of lipid and detergent indicated that the content of $\beta$-sheet secondary structure in hVDAC1 decreased at increased detergent content. Unlike bacterial $\beta$-barrel membrane proteins, hVDAC1 was not stable even in mild detergents such as LDAO or dodecylmaltoside. Spontaneous folding of outer membrane proteins into lipid bilayers indicates that in cells, the main purpose of membrane-inserted or associated assembly factors may be to select and target $\beta$-barrel membrane proteins towards the outer membrane instead of actively assembling them under consumption of energy as described for the translocons of cytoplasmic membranes.
\end{abstract}

Keywords: membrane protein folding; membrane protein stability; membrane insertion; outer membrane protein; VDAC

Abbreviations used: $\mathrm{C}_{8} \mathrm{E}_{4}$, tetraethyleneglycol-monooctylether; DDM, dodecylmaltoside; $d i \mathrm{C}_{n: 0} \mathrm{PC}, 1$,

2-diacyl-sn-glycero-3-phosphocholine (the acyl chain lengths range here from $n=10$ to $n=16$ carbons); $\operatorname{diC}_{18: 1} \mathrm{PC}, 1$, 2-dioleoyl-sn-glycero-3-phosphocholine; diPhPC, diphytanoyl phosphatidylcholine; FomA, major outer membrane protein of Fusobacterium nucleatum; hVDAC1, human isoform 1 of VDAC; IBs, inclusion bodies; LDAO, N-lauryl-N, $\mathrm{N}$-dimethylamine-N-oxide; LUVs, large unilamellar vesicles; NRMSD, normalized root mean square deviation; OMP, outer membrane protein; OmpA, outer membrane protein A of E. coli; PMSF, phenyl methane sulphonyl fluoride; TSS, transformation and storage solution; VDAC, voltage dependent anion selective channel.

E-mail address of the corresponding author: joerg.helmut.kleinschmidt@uni-konstanz.de 


\section{Introduction}

Membrane insertion and folding of transmembrane (TM) proteins (TMPs) has been studied mostly with bacterial membrane proteins, for example with the $\alpha$-helical bacteriorhodopsin (BR) of Halobacterium salinarium (see e.g. reviews ${ }^{1,2}$ and references therein) or with outer membrane protein A (OmpA) of Escherichia coli (see e.g. review ${ }^{3}$ and references therein), which forms a TM $\beta$-barrel domain. BR and OmpA belong to the two known classes of TMPs that are distinguished by their TM secondary structure. TM domains of cytoplasmic TMPs are helical while the outer membrane proteins (OMPs) of Gram-negative bacteria known to date exclusively form $\beta$-barrel TM domains. For $\alpha$-helix-bundle TMPs, a sequential three-stage model for insertion and folding has been described, while $\beta$-barrels insert and fold by a concerted mechanism. TMPs with $\beta-$ sheet secondary structure in their TM domain have also been reported for the outer membranes (OMs) of eukaryotic organelles. A prominent example is the voltage-dependent anion-selective channel (VDAC, 282 residues, $30.6 \mathrm{kDa}$ ) of the $\mathrm{OM}$ of mitochondria (see, e.g., for a review ${ }^{4}$ ). VDAC is synthesized in the cytosol and imported into mitochondria via the TOM complex. ${ }^{5}$ The human origin, the posttranslational insertion, and the presence of $\alpha$-helical structure with a lipid interface 6,7 in addition to the $\beta$-barrel TM domain make the human isoform 1 of VDAC (hVDAC1) an intriguing new model to explore the mechanism of insertion and folding of TMPs.

While VDACs are conserved among mammals (>98\% identity) there is much weaker sequence homology among VDACs of insects, plants, fungi, or yeast $(\sim 26 \%$ identity). VDAC is the major permeability pore of the mitochondrial OM with an inner diameter of $2.6 \mathrm{~nm}^{7}$ VDAC expression levels are critical for ATP synthesis capacity and cell growth. ${ }^{8}$ VDACs form a high conductance open state (4 to $4.5 \mathrm{nS}$ in $1 \mathrm{M} \mathrm{KCl}$ ) at small membrane potentials and switch to low conducting states ( 2 to $2.5 \mathrm{nS}$ ) at potentials $\geq 30 \mathrm{mV}$. $^{9}$

Single channel conductance recordings, in combination with streptavidin binding to biotinylated single cysteine mutants of VDAC that were reconstituted into lipid bilayers suggested that VDAC from Neurospora crassa consists of a single N-terminal TM $\alpha$-helix and a 13-stranded TM $\beta$-barrel. ${ }^{6,7}$ Both, the exact number of strands in the $\beta$-barrel domains of the various VDACs and the arrangement of the helix are a matter of debate. Alternatively, a 16stranded $\beta$-barrel has been proposed ${ }^{10,11}$ with the Nterminal amphipathic helix located predominantly at the membrane water-interface or outside the membrane. The topology may also depend on the species. Circular dichroism (CD) and infrared spectroscopic studies indicated that VDACs of various origins contain between 9 and $27 \% \alpha$-helical and between 30 and $53 \% \beta$-sheet structure. ${ }^{10,12,13}$ Bacterial porins of similar size like OmpF and FomA, contain only $\sim 2$ to $5 \%$ helical structure according to X-ray crystallography $(2 \mathrm{OMF})^{14}$ or CD spectroscopy. ${ }^{15}$ The majority of studies on the structure of VDAC have been performed in detergent micelles, while VDAC function was assayed in lipid bilayers."

Despite the necessity of proteinaceous folding factors in cells, several biochemical studies have shown that unfolded bacterial OMPs in $8 \mathrm{M}$ urea can spontaneously fold into detergent micelles to their active form upon urea dilution (for a review, see Buchanan ${ }^{16}$ ). The outer membrane proteins OmpA, ${ }^{17,18} \mathrm{OmpF}^{19}$ of $E$. coli and FomA of Fusobacterium nucleatum ${ }^{15}$ are the only $\beta$-barrel membrane proteins, for which folding of the urea-unfolded forms into lipid bilayers has been demonstrated, although attempts were also made with several other OMPs. Detergent micelles dissociate and reform fast, while lipid bilayers and biological membranes are tightly packed. Spontaneous insertion into lipid bilayers from an unfolded form, devoid of secondary structure, facilitates studies to decipher the rules of membrane protein folding.

Here we report that hVDAC1 can spontaneously insert and fold into lipid bilayers. Since, in contrast to bacterial OMPs, the hVDAC1 structure contains an additional $\alpha$-helix that may have a lipid interface, we examined the sequence by which elements of secondary structure are formed. We investigated folding of hVDAC1 into preformed bilayers of a range of phospholipids and compared it to folding of hVDAC1 into micelles of various detergents to determine whether the lipid bilayer affects hVDAC1 structure formation. To examine folding of hVDAC1, we analyzed the secondary structure of hVDAC1 in denatured form, in aqueous solution in the absence of lipid or detergent, after direct membrane insertion and folding, and after reconstitution from detergent micelles into lipid bilayers, including those of diphytanoyl phosphatidylcholine (diPhPC), the lipid widely used in functional studies of the single channel conductance of VDACs.

\section{Results}

\section{VDAC develops different secondary structure in detergents and in lipids}

hVDAC1 was expressed in form of inclusion bodies in E. coli, which were solubilized in urea. We first examined secondary structure formation in hVDAC1 by CD spectroscopy (Figure 1(a)). The spectra indicated that hVDAC1 was unfolded in $8 \mathrm{M}$ urea. After urea-dilution, CD spectra of aqueous hVDAC1 had one minimum at $\sim 214 \mathrm{~nm}$, demonstrating formation of $\beta$-sheet. The small amplitude and the line-shape of the spectrum indicated that the $\alpha$-helix content was negligible. After $\sim 18 \mathrm{~h}$ of dialysis, CD spectra of this form had slightly larger amplitudes. The composition of the secondary structure could not be determined, although we tried several deconvolution algorithms.

When urea solutions of hVDAC1 were diluted in the presence of detergent micelles, CD spectra of 


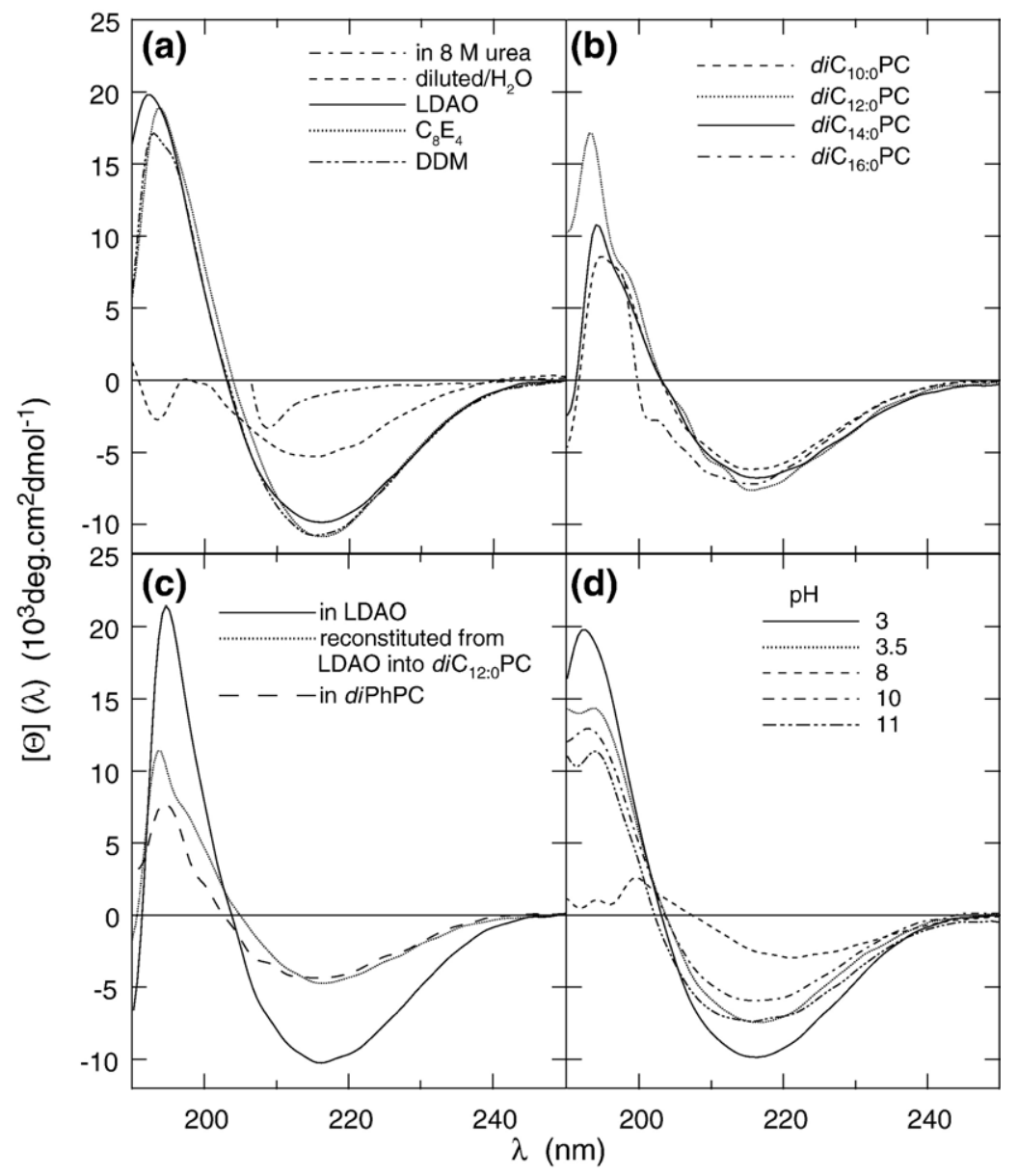

Figure 1. hVDAC1 forms a different secondary structure in lipid bilayers compared to detergents. (a) CD spectra of hVDAC1 in unfolded form in $8 \mathrm{M}$ urea $(-\cdot-)$, after 40-fold dilution of urea in aqueous solution $(--)$ and after incubation with micelles of LDAO $(-), \mathrm{C}_{8} \mathrm{E}_{4}(\cdots)$, or DDM $(-\cdots)$. (b) CD spectra of hVDAC1 in LUVs of $\operatorname{diC}_{10: 0} \mathrm{PC}(--), \operatorname{diC}_{12: 0} \mathrm{PC}(\cdot \cdots)$, $d^{2} \mathrm{C}_{14: 0} \mathrm{PC}(-)$, and of $d i \mathrm{C}_{16: 0} \mathrm{PC}$ $(-\cdot-)$. (c) CD spectra of hVDAC1, either incubated in LDAO micelles $(-)$, incubated in LDAO and then folded into $\mathrm{diC}_{12: 0} \mathrm{PC}$ vesicles $(\cdots)$ or incubated in LDAO and reconstituted into diPhPC (- -). (d) CD spectra of hVDAC1 in LDAO at $\mathrm{pH}$ $3(-), 3.5(\cdots), 8(--), 10(-\cdot-)$ and $11(-\cdots)$. For normalized CD spectra, mean residue molar ellipticities, $[\Theta]$ $(\lambda)$, were calculated as described in Materials and Methods.

hVDAC1 had much larger amplitudes (Figure 1(a)). As detergents, $N$-lauryl- $N$, N-dimethylamine- $N$ oxide (LDAO), tetraethyleneglycol monooctylether $\left(\mathrm{C}_{8} \mathrm{E}_{4}\right)$, or dodecylmaltoside (DDM) were used and resulted in very similar hVDAC1 spectra. Between pH 4 and 9, hVDAC1 (theoretical pI 8.6) partially precipitated and good solubility was obtained at $\mathrm{pH} 3$ (Figure $1(\mathrm{~d})$ ). Protonation at $\mathrm{pH} 3$ results in a positive net charge and increases solubility, which in cells is conferred by molecular chaperones. CD spectra of hVDAC1 in detergent were characteristic of predominantly $\beta$-sheet structure and were very similar to previously published spectra of VDAC of $N$. crassa isolated in native form in octyl- $\beta$-glucoside at $\mathrm{pH} 7{ }^{13}$ Spectral deconvolution indicated formation of $\sim 16 \% \alpha$-helical, $\sim 32 \% \beta$-strand, and $\sim 23 \%$ $\beta$-turn structure for hVDAC1 in detergent micelles, averaged over hVDAC1 samples in all mild detergents (Table 1).

To investigate folding of hVDAC1 into phospholipid membranes, we prepared large unilamellar vesicles (LUVs) of phosphatidylcholines (PC) with two saturated acyl chains of different hydrophobic length $n\left(d i \mathrm{C}_{\mathrm{n}: 0}\right)$, namely $d i \mathrm{C}_{10: 0} \mathrm{PC}, \mathrm{diC}_{11: 0} \mathrm{PC}$, $d i C_{12: 0} P C, d i C_{13: 0} P C$, and $d i C_{14: 0} P C$. hVDAC1 folding was initiated by urea-dilution and CD spectra were recorded (Figure 1(b)). The CD spectra of hVDAC1 in these lipids were very similar and also indicated predominantly $\beta$-sheet structure, but compared to spectra of hVDAC1 in detergents, their amplitudes were lower. Consistent with this observation, deconvolution analysis showed lower $\alpha$-helix $(\sim 10.5 \%)$ and higher $\beta$-strand $(36.5 \%)$ content in hVDAC1 in phospholipids (Table 2) in comparison to hVDAC1 in detergents (Table 1). For samples of hVDAC1 in $d i \mathrm{C}_{18: 1} \mathrm{PC}$ and $d i \mathrm{C}_{16: 0} \mathrm{PC}$, deconvolution analyses of the CD spectra were not very accurate, because of increased light scattering and decreased signal/noise ratios at $\lambda<210 \mathrm{~nm}$. hVDAC1 in thin $d i \mathrm{C}_{10: 0} \mathrm{PC}$ bilayers had a lower $\beta$ strand content, but did not display an increased $\alpha$ helix content.

When hVDAC1 was first solubilized in LDAO micelles and then folded into $d i \mathrm{C}_{12: 0} \mathrm{PC}$, the $\mathrm{CD}$ spectra matched the spectra of hVDAC1 directly folded into $d i \mathrm{C}_{12: 0} \mathrm{PC}$ bilayers in the absence of detergent (Figure 1(c)). The analysis revealed similar contents of $\alpha$-helix and $\beta$-sheet (Table 2). Obviously, hVDAC1 develops different secondary structure in detergent micelles and in lipid bilayers.

\section{Proteolysis leads to different fragmentation of hVDAC1 in bilayers and in micelles}

In water, hVDAC1 was completely cleaved within $30 \mathrm{~min}$ by the protease pepsin (Figure 2). In LDAO, however, pepsinolysis was incomplete within the first $30 \mathrm{~min}$ and multiple fragments were obtained (Figure 2(b) lanes 3, 5, 7, and 9). The largest fragments were observed at $\sim 27 \mathrm{kDa}$ and at $\sim 24 \mathrm{kDa}$. 
Table 1. Analysis of the CD spectra of hVDAC1 in detergent micelles

\begin{tabular}{|c|c|c|c|c|c|c|}
\hline Sample & Algorithm & $\alpha$-Helix (\%) & $\beta$-Strand $(\%)$ & $\beta$-Turns (\%) & Random coil (\%) & NRMSD $^{a}$ \\
\hline \multirow[t]{4}{*}{ LDAO (Sample 1) } & SELCON3 & 16.5 & 33.7 & 22.8 & 26.9 & 0.15 \\
\hline & CONTIN & 16.2 & 33.5 & 23.4 & 27 & 0.08 \\
\hline & CDSSTR & 14.1 & 32.3 & 23.2 & 30.3 & 0.04 \\
\hline & Average & 15.6 & 33.2 & 23.1 & 28.1 & \\
\hline \multirow[t]{4}{*}{ LDAO (Sample 2) } & SELCON3 & 16.9 & 31.4 & 22.5 & 29.3 & 0.12 \\
\hline & CONTIN & 14.4 & 30.3 & 24.1 & 30.7 & 0.05 \\
\hline & CDSSTR & 12.8 & 32.1 & 23 & 32 & 0.02 \\
\hline & Average & 14.7 & 31.3 & 23.2 & 30.7 & \\
\hline \multirow[t]{4}{*}{ LDAO (Sample 3) } & SELCON3 & 16.9 & 30.7 & 22.5 & 28.9 & 0.14 \\
\hline & CONTIN & 15.4 & 31 & 22.9 & 30.7 & 0.03 \\
\hline & CDSSTR & 13 & 33 & 23 & 31 & 0.01 \\
\hline & Average & 15.1 & 31.5 & 22.8 & 30.2 & \\
\hline \multirow{4}{*}{ LDAO (Sample 4) } & SELCON3 & 21 & 28 & 22.7 & 28.0 & 0.16 \\
\hline & CONTIN & 17.7 & 29.5 & 23.2 & 29.5 & 0.06 \\
\hline & CDSSTR & 14.8 & 30.7 & 24 & 31 & 0.03 \\
\hline & Average & 17.8 & 29.4 & 23.3 & 29.5 & \\
\hline \multirow[t]{4}{*}{ LDAO (Sample 5) } & SELCON3 & 14.5 & 33.6 & 22.9 & 28.7 & 0.16 \\
\hline & CONTIN & 14.8 & 34.2 & 22.9 & 28 & 0.07 \\
\hline & CDSSTR & 13.3 & 33.4 & 23 & 30 & 0.03 \\
\hline & Average & 14.2 & 33.7 & 22.9 & 28.9 & \\
\hline \multirow[t]{4}{*}{ LDAO (average of 5 samples) } & SELCON3 & $17.2 \pm 2.3$ & $30.7 \pm 2.3$ & $22.5 \pm 0.17$ & $28.9 \pm 0.9$ & \\
\hline & CONTIN & $15.7 \pm 1.3$ & $31.7 \pm 2.0$ & $23.3 \pm 0.49$ & $29.2 \pm 1.6$ & \\
\hline & CDSSTR & $13.6 \pm 0.8$ & $32.3 \pm 1.0$ & $23.2 \pm 0.4$ & $30.9 \pm 0.77$ & \\
\hline & Average & 15.5 & 31.5 & 23 & 29.7 & \\
\hline \multirow[t]{4}{*}{ DDM } & SELCON3 & 24.5 & 25.8 & 23.5 & 26.1 & 0.16 \\
\hline & CONTIN & 17.8 & 34.4 & 21.1 & 26.5 & 0.13 \\
\hline & CDSSTR & 14.6 & 33.3 & 24 & 28 & 0.04 \\
\hline & Average & 18.9 & 31.2 & 22.8 & 26.8 & \\
\hline \multirow[t]{4}{*}{$\mathrm{C}_{8} \mathrm{E}_{4}$} & SELCON3 & 24.6 & 28.5 & 23.4 & 26.1 & 0.19 \\
\hline & CONTIN & 15 & 37.8 & 23.8 & 23.4 & 0.13 \\
\hline & CDSSTR & 13.7 & 35.8 & 23 & 27 & 0.03 \\
\hline & Average & 17.7 & 34 & 23.3 & 25.5 & \\
\hline \multirow[t]{4}{*}{ SDS } & SELCON 3 & 27.5 & 19.1 & 21.8 & 27.2 & 0.17 \\
\hline & CONTIN & 29.1 & 22.7 & 22.2 & 26 & 0.17 \\
\hline & CDSSTR & 30.7 & 26.4 & 19 & 24 & 0.02 \\
\hline & Average & 29.1 & 24.6 & 21.0 & 25.7 & \\
\hline \multirow[t]{4}{*}{ 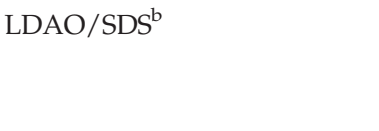 } & SELCON 3 & 29 & 19.3 & 21.6 & 27.3 & 0.15 \\
\hline & CONTIN & 31 & 20.1 & 23.5 & 25.4 & 0.13 \\
\hline & CDSSTR & 33.8 & 22.2 & 20 & 23 & 0.02 \\
\hline & Average & 31.3 & 20.5 & 21.7 & 25.2 & \\
\hline
\end{tabular}

The composition of the secondary structure of hVDAC1 in the different samples was analyzed from CD spectra using DICHROWEB ${ }^{38}$ and the algorithms CONTIN, ${ }^{39}$ SELCON3,${ }^{40}$ and CDSSTR. ${ }^{41}$

${ }^{a}$ NRMSD is the normalized root mean square deviation obtained from the analysis and should be $\sim 0.25$ or below. Results with a higher NRMSD are given in italics and were listed when different algorithms were in agreement.

${ }^{b}$ hVDAC1 was incubated in LDAO, then diluted below its CMC in the presence of SDS prior to dialysis.

Similar fragments were obtained by proteolysis of hVDAC1 that was solubilized in either DDM or $\mathrm{C}_{8} \mathrm{E}_{4}$ (data not shown). Pepsinolysis was complete after $1 \mathrm{~h}$.

Pepsinolysis for $4 \mathrm{~h}$ of folded hVDAC1 in lipid bilayers of either $d i \mathrm{C}_{10: 0} \mathrm{PC}, d i \mathrm{C}_{11: 0} \mathrm{PC}, d i \mathrm{C}_{12: 0} \mathrm{PC}$, $d i \mathrm{C}_{13: 0} \mathrm{PC}$, or $d i \mathrm{C}_{14: 0} \mathrm{PC}$ at $\mathrm{pH} 3$ resulted in a single fragment of $\sim 27 \mathrm{kDa}$, as depicted for $d i \mathrm{C}_{12: 0} \mathrm{PC}$ (Figure 2(b), lanes 4, 6, 8, 13, 15, and 17). The $27 \mathrm{kDa}$ fragment remained protected even at prolonged times of proteolysis. When inserted into lipid bilayers, only $\sim 37$ residues, $\mathrm{N}$-terminal or $\mathrm{C}$-terminal, were proteolytically cleaved. A single band was observed after pepsinolysis, indicating oriented insertion of hVDAC1 into lipid bilayers with the cleavage-site for pepsin located always outside the lipid vesicle.

\section{hVDAC1 folds into its active form in lipid bilayers}

For single channel recordings, hVDAC1 in LDAO was reconstituted into black diPhPC lipid membranes and a voltage of $U=10 \mathrm{mV}$ was applied across the membrane. Addition of LDAO alone, i.e. in the absence of hVDAC1, did not result in conductance across the lipid bilayer, confirming 
Table 2. Analysis of the CD spectra of hVDAC1 in lipid bilayers

\begin{tabular}{|c|c|c|c|c|c|c|}
\hline Sample & Algorithm (\%) & $\alpha$-Helix (\%) & $\beta$-Strand $(\%)$ & $\beta$-Turns (\%) & Random coil (\%) & NRMSD $^{\mathrm{a}}$ \\
\hline \multirow[t]{4}{*}{$\mathrm{diC}_{10: 0} \mathrm{PC}$} & SELCON3 & 12.0 & 35.4 & 24.1 & 28.9 & 0.40 \\
\hline & CONTIN & 10.3 & 30.5 & 25 & 34.2 & 0.22 \\
\hline & CDSSTR & 14.2 & 29.4 & 26 & 31 & 0.06 \\
\hline & Average & 12.1 & 31.8 & 25 & 31.3 & \\
\hline \multirow[t]{4}{*}{$d_{i 1} \mathrm{C}_{11: 0} \mathrm{PC}$} & SELCON3 & 12.3 & 37.1 & 19.1 & 30.7 & 0.15 \\
\hline & CONTIN & 10.5 & 37.4 & 22.1 & 29.9 & 0.11 \\
\hline & CDSSTR & 9.7 & 37.2 & 22 & 30 & 0.05 \\
\hline & Average & 10.8 & 37.2 & 21 & 30.2 & \\
\hline \multirow[t]{4}{*}{$d i \mathrm{C}_{12: 0} \mathrm{PC}(\mathrm{I} .)^{\mathrm{b}}$} & SELCON3 & 11.3 & 36.6 & 22.6 & 29.6 & 0.16 \\
\hline & CONTIN & 11.8 & 36.3 & 22.4 & 29.5 & 0.12 \\
\hline & CDSSTR & 11 & 35 & 23 & 31 & 0.03 \\
\hline & Average & 11.4 & 36.0 & 22.7 & 30.0 & \\
\hline \multirow[t]{4}{*}{$d i \mathrm{C}_{12: 0} \mathrm{PC}(\mathrm{II} .)^{\mathrm{b}}$} & SELCON3 & 7.9 & 37.5 & 24.8 & 28.6 & 0.35 \\
\hline & CONTIN & 9.6 & 38.7 & 23.5 & 28.2 & 0.27 \\
\hline & CDSSTR & 11.4 & 36 & 27 & 27 & 0.07 \\
\hline & Average & 9.6 & 37.4 & 25.1 & 27.9 & \\
\hline \multirow[t]{4}{*}{$\mathrm{diC}_{13: 0} \mathrm{PC}$} & SELCON3 & 10.8 & 37.2 & 23.5 & 28.7 & 0.26 \\
\hline & CONTIN & 11.5 & 39.7 & 22.5 & 26.3 & 0.14 \\
\hline & CDSSTR & 11.1 & 32.3 & 25 & 32 & 0.08 \\
\hline & Average & 11.1 & 36.4 & 23.6 & 29 & \\
\hline \multirow[t]{4}{*}{$d i \mathrm{C}_{14: 0} \mathrm{PC}$} & SELCON3 & 10.4 & 36 & 21.5 & 32 & 0.29 \\
\hline & CONTIN & 10.2 & 35.1 & 22.2 & 32.4 & 0.21 \\
\hline & CDSSTR & 7.1 & 36.4 & 23.2 & 33.3 & 0.10 \\
\hline & Average & 9.2 & 35.8 & 22.3 & 32.6 & \\
\hline \multirow[t]{4}{*}{$d i \mathrm{PhPC}^{\mathrm{b}}$} & SELCON3 & 15.8 & 41.3 & 10.2 & 17.3 & 0.25 \\
\hline & CONTIN & 7.7 & 37.3 & 22.6 & 32.4 & 0.17 \\
\hline & CDSSTR & 9.5 & 35.1 & 23 & 32 & 0.06 \\
\hline & Average & 8.6 & 36.2 & 22.8 & 32.2 & \\
\hline
\end{tabular}

The composition of the secondary structure of hVDAC1 in the different samples was analyzed as described in the legend to Table 1.

a NRMSD is the normalized root mean square deviation obtained from the analysis and should be $\sim 0.25$ or below. Results with a higher NRMSD are given in italics and were listed when different algorithms were in agreement.

b Analysis I was performed on hVDAC1 directly folded into di $\mathrm{C}_{12: 0} \mathrm{PC}$ (the CD spectrum is shown in Figure $1(\mathrm{~b})$ ). Analysis II was performed on hVDAC1 that was first solubilized in LDAO and then reconstituted into diC $\mathrm{C}_{12: 0} \mathrm{PC}$ (the CD spectrum is shown in Figure 1(c)).

previous results. ${ }^{15}$ Upon addition of hVDAC1 in LDAO, a stepwise increase of the current across the lipid bilayer was observed (Figure 3(a), inset) and
$50 \%$ of the corresponding channel events had a conductance of $4.5( \pm 0.5) \mathrm{nS}$, indicating insertion of single channels. For a single channel opening, a
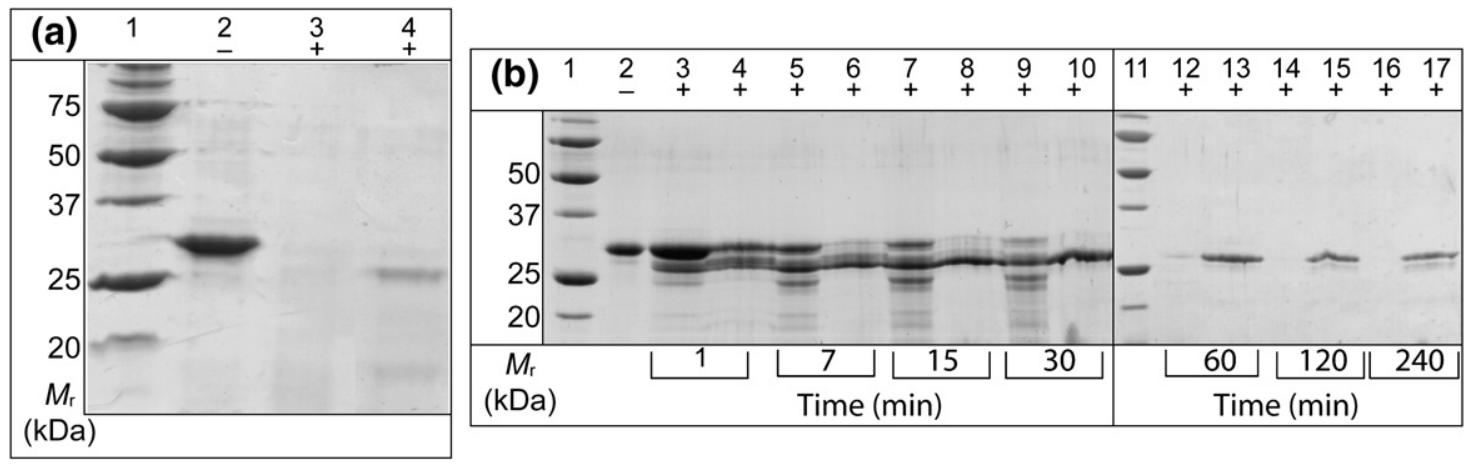

Figure 2. Pepsinolysis at $\mathrm{pH} 3$ and analysis by SDS-PAGE demonstrates that hVDAC1 inserts into micelles or lipid bilayers. Protein standards are shown in lanes 1 and 11. (a) Unfolded hVDAC1 in urea migrates at $30 \mathrm{kDa}$ (lane 2). Aqueous hVDAC1 is completely cleaved by pepsin (indicated by +) within $30 \mathrm{~min}$ (lane 3 ). After folding of hVDAC1 into $\mathrm{diC}_{14: 0} \mathrm{PC}$, pepsinolysis is incomplete and leads to a fragment with a size of $\sim 26-27 \mathrm{kDa}$ (lane 4) after $240 \mathrm{~min}$. (b) Pepsinolysis of hVDAC1 in LDAO (lanes $3,5,7,9,12,14,16$ ), and in di $_{12: 0} \mathrm{PC}$ (lanes 4, 6, 8, 10, 13, 15, 17), indicate

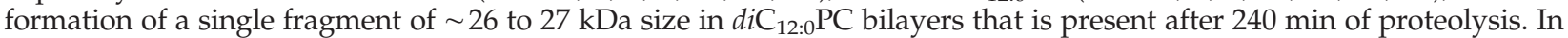
LDAO, hVDAC1 is first cleaved into two major fragments of $\sim 26$ to $27 \mathrm{kDa}$ and of $\sim 24 \mathrm{kDa}$. Additional fragments of weaker intensity were also observed. Pepsinolysis of hVDAC1 in LDAO is complete after $60 \mathrm{~min}$. 

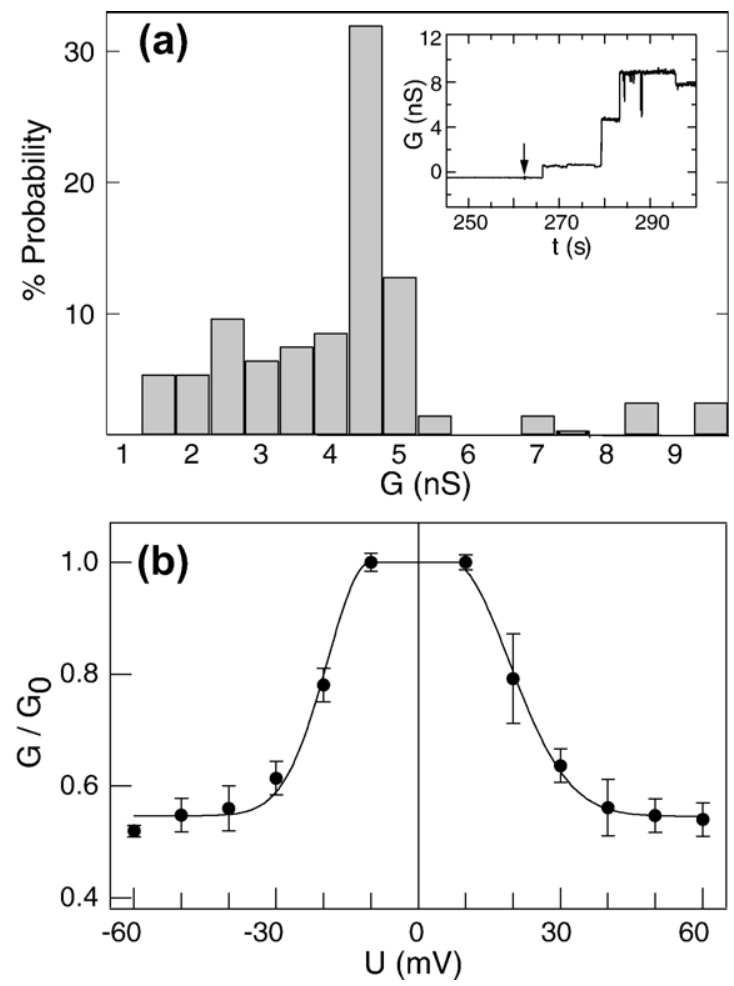

Figure 3. Single channel recordings demonstrate function of folded hVDAC1. (a) The histogram of hVDAC1 conductance indicates an average single channel conductance of $G_{0}=4.5 \mathrm{nS}$ at $10 \mathrm{mV}$ for hVDAC1 in $1 \mathrm{M} \mathrm{KCl} .94$ single channel events were recorded at RT. The inset shows the stepwise increase in conductance across black lipid films of diPhPC at $\mathrm{pH} 7$, upon addition (marked by $\downarrow$ ) of hVDAC1 solubilized in LDAO. (b) The conductance, $G$, of hVDAC1 is voltage-dependent. Above $10 \mathrm{mV}$, hVDAC1 forms channels of smaller conductance. Relative conductance, $G / G_{0}$, is plotted as a function of the applied voltage.

conductance of $G_{0}=4.0$ to $4.5 \mathrm{nS}$ at $10 \mathrm{mV}$ in $1 \mathrm{M} \mathrm{KCl}$ was most frequent (Figure 3(a)), in agreement with the conductance of natively isolated hVDAC1 from membranes ${ }^{20}$ and other natively isolated VDAC channels from various organisms. ${ }^{21-23}$ At absolute voltages $|U|>10 \mathrm{mV}$, up to $|U|=30 \mathrm{mV}$, conductance decreased steeply. hVDAC1 switched to states of lower conductance with $G \approx 0.5 G_{0}$ (Figure $3(b)$ ). Very similar voltage-gating was previously observed for hVDAC1 that was isolated from membranes in native form. ${ }^{9,20}$

The analysis of CD spectra of hVDAC1 after reconstitution into diPhPC membranes used in these functional studies indicated the same secondary structure as of hVDAC1 directly folded into bilayers of different $d i \mathrm{C}_{\mathrm{n}: 0} \mathrm{PC}$ phospholipids (Figure 1(c), Table 2). It was, however, not possible to fold ureaunfolded hVDAC1 directly into diPhPC without first solubilizing it in LDAO.

\section{hVDAC1 folds into lipid bilayers with high yield}

To analyze homogeneity and folding yields of hVDAC1 in bilayers of $d_{i C_{12: 0}} \mathrm{PC}$, we performed sucrose density gradient centrifugation. Two layers containing hVDAC1 were obtained. The layer at higher density (HD) contained the folded hVDAC1 (Figure 4(a)), while the layer of lower density (LD) indicated complete absence of $\beta$-sheets or $\alpha$-helices in CD spectroscopy. 92\% of hVDAC1 was folded, since $0.23 \mathrm{mg}$ were found in the HD layer and $0.02 \mathrm{mg}$ in the LD layer, using the assay described by Lowry et al. ${ }^{24}$ Similarly, 94\% folded hVDAC1 were estimated by densitometry from the polyacrylamide gel of the two layers (Figure 4(a) inset). Phosphate analysis indicated a molar lipid/folded hVDAC1 ratio of 450 .

\section{pH alteration strongly affects hVDAC1 structure in detergent but not in lipid bilayers}

We examined the secondary structure of hVDAC1 in micelles and in bilayers after increasing the $\mathrm{pH}$ from 3 to 7 . While adjustment to $\mathrm{pH} 7$ had little effect on the secondary structure of hVDAC1 in $d_{i 2} \mathrm{C}_{12: 0} \mathrm{PC}$ bilayers (Figure 4(b)) or diPhPC (not shown), the spectrum of hVDAC1 in LDAO displayed both, a strong reduction in amplitude and a shift of the spectral minimum from $216 \mathrm{~nm}$ to $220 \mathrm{~nm}$. Increased

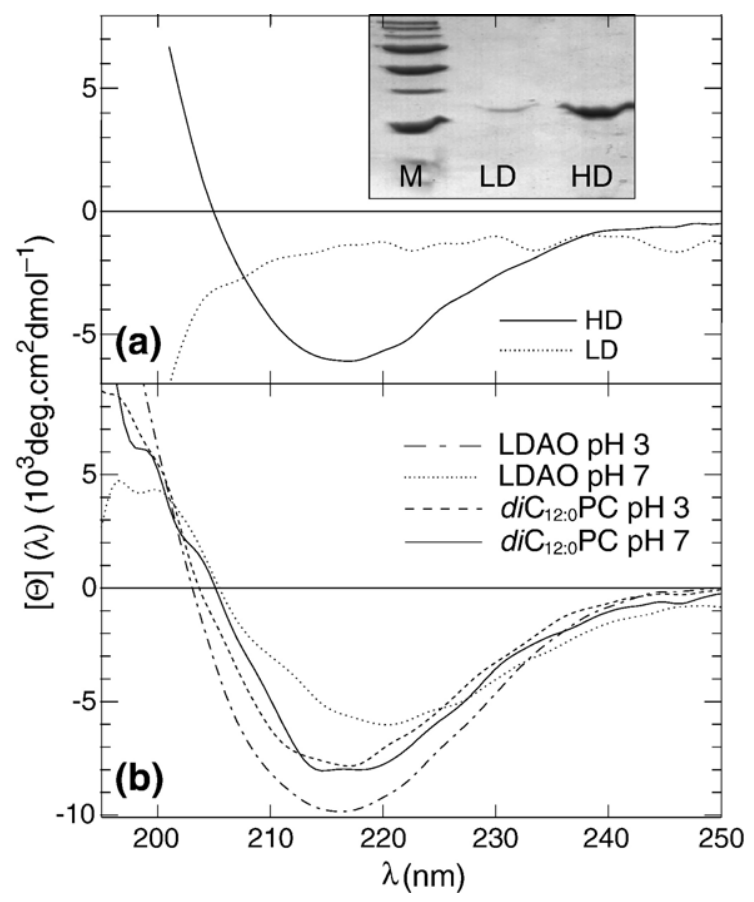

Figure 4. (a) Folding of hVDAC1 into lipid bilayers is near quantitative. Sucrose density gradient centrifugation of folded hVDAC1 in $d_{i C_{12: 0}} \mathrm{PC}$ resulted in two layers of lower (LD) and higher density (HD). The HD layer contained more than $92 \%$ of hVDAC1. The CD spectra indicated hVDAC1 was folded in the HD (-), but not in the LD $(\cdots)$ layer. Inset: SDS-PAGE analysis of both layers, with molecular weight markers $(M)$. (b) The CD spectra of hVDAC1 in LDAO micelles at $\mathrm{pH} 3(-\cdot-)$ and immediately after adjustment of the $\mathrm{pH}$ to $7(\cdots)$ indicated a strong conformational change upon $\mathrm{pH}$ adjustment, while hVDAC1 spectra in $d i \mathrm{C}_{12: 0} \mathrm{PC}$ bilayers were very similar at $\mathrm{pH} 3(--)$ and at $\mathrm{pH} 7$ (-). 


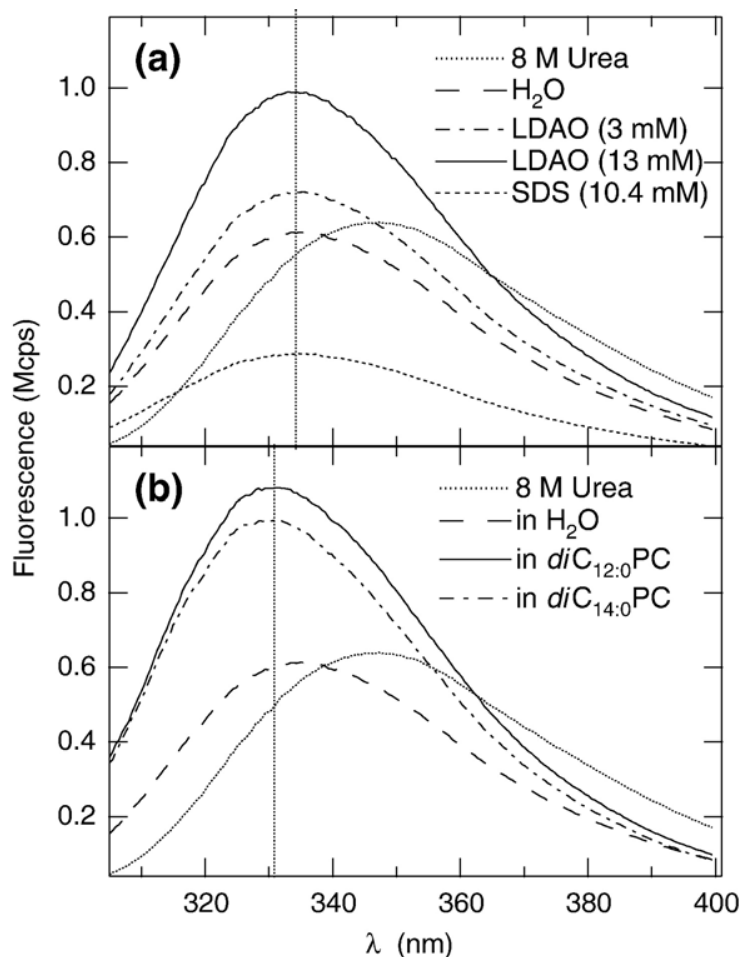

Figure 5. Fluorescence spectroscopy indicates a different microenvironment of the tryptophan residues of hVDAC1 in LDAO micelles and in lipid bilayers. (a) The fluorescence spectra of $1.3 \mu \mathrm{M}$ hVDAC1 in $3 \mathrm{mM}$ LDAO $(-\cdot-)$, in $13 \mathrm{mM} \mathrm{LDAO}(-)$, and in $10.4 \mathrm{mM}$ SDS $(---)$ have maxima at $335 \mathrm{~nm}$. (b) The fluorescence spectra of $\mathrm{hVDAC} 1$ in an 800-fold molar excess of $d i \mathrm{C}_{12: 0} \mathrm{PC}(-)$ or $d i \mathrm{C}_{14: 0} \mathrm{PC}\left(-^{\cdot-}\right)$ have maxima at $330 \mathrm{~nm}$. Also shown are fluorescence spectra of unfolded hVDAC1 in $8 \mathrm{M}$ urea $(\cdots)$ and of aqueous hVDAC1 after urea dilution $(--)$.

incubation time did not alter spectra of the lipid bilayer-inserted form, while the spectral amplitude of the LDAO solubilized form decreased further.

\section{Fluorescence spectroscopy indicated hVDAC1 insertion into bilayers and micelles}

Fluorescence spectra of unfolded hVDAC1 in urea had a maximum at $347 \mathrm{~nm}$ (Figure 5). After urea dilution, the maximum was shifted to $335 \mathrm{~nm}$. Compared to this aqueous form, the fluorescence maximum of hVDAC1 in $13 \mathrm{mM}$ LDAO (Figure 5(a)) displayed only a small blue shift to $334 \mathrm{~nm}$, but fluorescence intensity was much higher. The same wavelength of the maximum was observed in $0.3 \%$ SDS, but fluorescence was strongly decreased. In comparison, a much stronger blue shift was observed for spectra of hVDAC1 in lipid bilayers of $d i \mathrm{C}_{12: 0} \mathrm{PC}$ or $\mathrm{diC}_{14: 0} \mathrm{PC}$, with a fluorescence maximum at $330 \mathrm{~nm}$ (Figure 5(b)). Fluorescence of hVDAC1 in lipid bilayers was most intense. The more pronounced blue shift indicated that the average microenvironment of the tryptophan residues of hVDAC1 in lipid was more hydrophobic than in LDAO.

\section{hVDAC1 secondary structure in mixtures of LDAO detergent with diC $_{12: 0} \mathrm{PC}$}

CD spectra, fluorescence spectra, and limited proteolysis of $\mathrm{hVDAC} 1$ demonstrated that hVDAC1 is not natively inserted and folded in detergent micelles. To determine whether the change in hVDAC1 secondary structure is determined by the presence of a lipid or by the supramolecular structure, i.e. micelles or bilayers, we incubated hVDAC1 in mixtures of different $\mathrm{LDAO} / d i \mathrm{C}_{12: 0} \mathrm{PC}$ ratios and determined the composition of the secondary structure of hVDAC1 in these mixtures by recording CD spectra (Figure 6). The amplitudes of the mean residue molar ellipticities of the $\mathrm{CD}$ spectra between 190 and $200 \mathrm{~nm}$ and between 210 and $220 \mathrm{~nm}$ increased at higher LDAO content, indicating an increase in the fraction of $\alpha$ - helix structure at higher $\mathrm{LDAO}$ content in $\mathrm{LDAO} / \mathrm{diC}_{12: 0} \mathrm{PC}$ mixtures. Similarly, at increased content of LDAO, the maximum of the spectrum shifted from $\lambda \approx 195 \mathrm{~nm}$ to $\lambda \approx 192 \mathrm{~nm}$, indicating more $\alpha$-helical structure. The compositions of the secondary structure of hVDAC 1 at the various detergent/lipid ratios were obtained by spectral deconvolution and are given in Table 3.

The percentage of $\alpha$-helix $(O)$ increased while the percentage $\beta$-sheet ( $\square$ ) secondary structure of hVDAC1 decreased with increased mole fraction of LDAO (Figure 7), indicating that the presence of only a few lipid molecules per protein molecule is insufficient to stabilize the native hVDAC1 structure. Instead, the replacement of phospholipids by LDAO at the surface of hVDAC1 results in a lower fraction of $\beta$-sheet secondary structure.

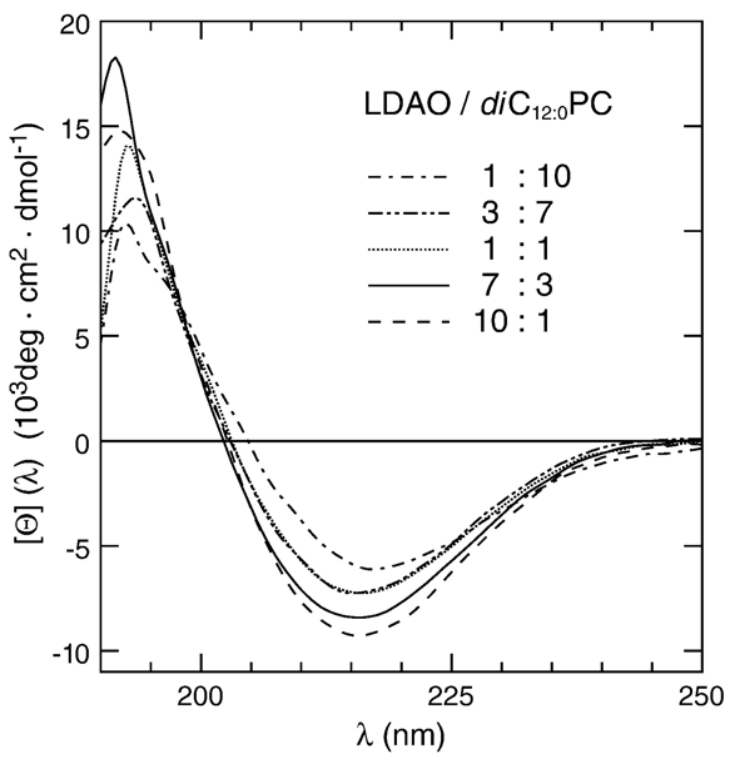

Figure 6. Normalized CD spectra of hVDAC1 in detergent/lipid mixed micelles of LDAO and $d i \mathrm{C}_{12: 0} \mathrm{PC}$. The increase in the spectral amplitude and the shift in the maximum of the spectra from $\lambda \approx 195 \mathrm{~nm}$ to $\lambda \approx 192 \mathrm{~nm}$ is characteristic for an increase in the $\alpha$-helical fraction at higher $\mathrm{LDAO}$ content in $\mathrm{LDAO} / \mathrm{diC}_{12: 0} \mathrm{PC}$ mixtures. 
Table 3. Analysis of the CD spectra of hVDAC1 in lipid/detergent mixtures

\begin{tabular}{|c|c|c|c|c|c|c|}
\hline Sample & Algorithm & $\alpha$-Helix (\%) & $\beta$-Strand $(\%)$ & $\beta$-Turns $(\%)$ & Random coil (\%) & NRMSD $^{\mathrm{a}}$ \\
\hline \multirow[t]{4}{*}{$\mathrm{LDAO} / \mathrm{diC}_{12: 0} \mathrm{PC} 1: 10$} & SELCON 3 & 10.9 & 38.0 & 21.8 & 27.5 & 0.18 \\
\hline & CONTIN & 12.1 & 37.6 & 22.5 & 25.4 & 0.14 \\
\hline & CDSSTR & 10.8 & 35.3 & 23 & 31 & 0.06 \\
\hline & Average & 11.3 & 37 & 22.4 & 29 & \\
\hline \multirow[t]{4}{*}{$\mathrm{LDAO} / \mathrm{diC}_{12: 0} \mathrm{PC} 3: 7$} & SELCON 3 & 13.1 & 35.7 & 22.2 & 28.4 & 0.16 \\
\hline & CONTIN & 12.2 & 35.6 & 22.5 & 29.8 & 0.08 \\
\hline & CDSSTR & 11.2 & 34.2 & 23 & 32 & 0.02 \\
\hline & Average & 12.2 & 35.1 & 22.5 & 30 & \\
\hline \multirow{4}{*}{$\mathrm{LDAO} / d i \mathrm{C}_{12: 0} \mathrm{PC} 1: 1$} & SELCON 3 & 13.5 & 35.3 & 22.8 & 27.5 & 0.17 \\
\hline & CONTIN & 13.8 & 35.4 & 24.3 & 26.6 & 0.12 \\
\hline & CDSSTR & 10.7 & 36.9 & 22 & 30 & 0.06 \\
\hline & Average & 12.7 & 35.8 & 23 & 28 & \\
\hline \multirow[t]{4}{*}{$\mathrm{LDAO} / \mathrm{diC}_{12: 0} \mathrm{PC} 7: 3$} & SELCON 3 & 16.5 & 33.9 & 21.1 & 29 & 0.21 \\
\hline & CONTIN & 13.1 & 34.5 & 22.6 & 29.8 & 0.09 \\
\hline & CDSSTR & 9.2 & 36.4 & 23 & 31 & 0.02 \\
\hline & Average & 12.9 & 34.9 & 22.2 & 29.9 & \\
\hline \multirow[t]{4}{*}{$\mathrm{LDAO} / \mathrm{diC}_{12: 0} \mathrm{PC} 10: 1$} & SELCON 3 & 17.5 & 31.4 & 21.9 & 29.1 & 0.18 \\
\hline & CONTIN & 14.6 & 33 & 22.2 & 30.3 & 0.04 \\
\hline & CDSSTR & 11.6 & 34.4 & 23 & 31 & 0.01 \\
\hline & Average & 14.6 & 32.9 & 22.4 & 30.1 & \\
\hline
\end{tabular}

The composition of the secondary structure was analyzed as described in the legend to Table 1.

a NRMSD is the normalized root mean square deviation obtained from the analysis and should be $\sim 0.25$ or below.

\section{SDS irreversibly denatures hVDAC1 at room temperature}

Denatured and folded forms of many OMPs of Gram-negative bacteria can be distinguished by their migration on sodium dodecyl sulfate polyacrylamide (SDS-PAGE) gels, if the samples are not boiled prior to electrophoresis (see, e.g. Kleinschmidt ${ }^{3}$ and references therein). Folded, solubilized and unfolded forms of hVDAC1 did not show any differences in electrophoretic mobility, neither in SDS-PAGE nor in native gel ${ }^{25}$ analysis. Since the electrophoresis results suggested that the $\beta$-barrel structure of hVDAC1 is

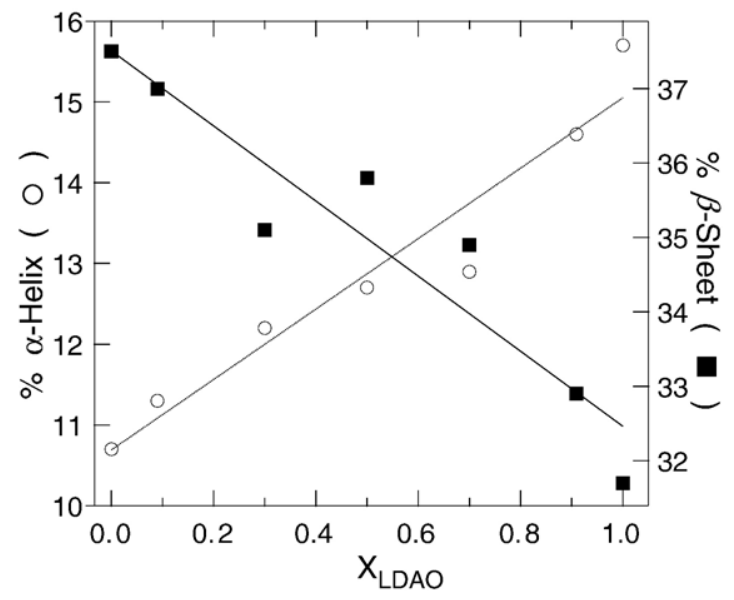

Figure 7. The contents of $\alpha$-helix $(O)$ and $\beta$-sheet $(\boldsymbol{\square})$ in hVDAC1, derived from CD spectra (Figure 6 and Table $3)$, are a function of the molar fraction of LDAO in binary mixtures with $d i \mathrm{C}_{12: 0} \mathrm{PC}$. The molar amphiphile (LDAO+ $\left.d_{i 2} \mathrm{C}_{12: 0} \mathrm{PC}\right) / \mathrm{hVDAC1}$ ratio was 800 . not very stable, we recorded CD spectra of hVDAC1 in $0.3 \%$ SDS after urea-dilution (Figure 8 , broken line). In a second experiment, we solubilized hVDAC1 into LDAO detergent micelles and then diluted LDAO below its CMC in the presence of $0.3 \%$ SDS, dialyzed the sample in $0.3 \%$ SDS and recorded the CD spectrum (Figure 8, dotted line). Spectra of both samples were very similar and had a secondary structure composition of $\sim 30 \% \alpha$-helix and $\sim 21 \%$ $\beta$-sheet (Table 1). This is a twofold increase in $\alpha$-helix

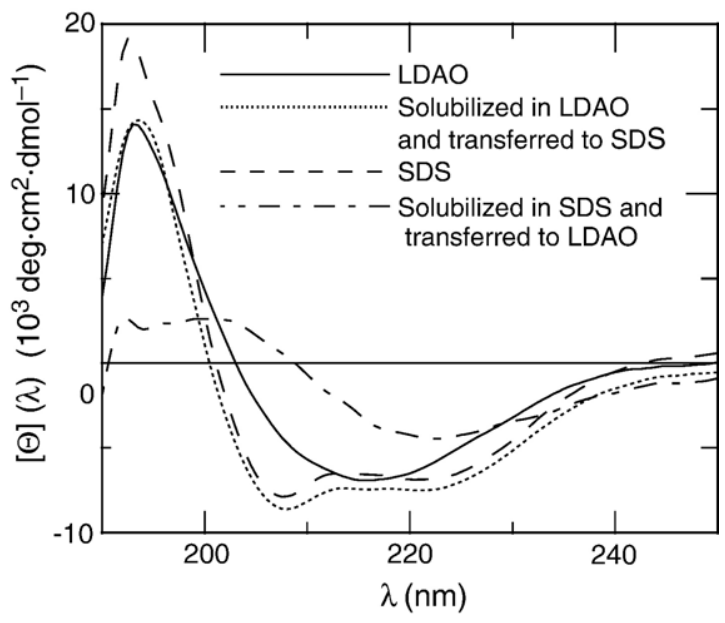

Figure 8. CD spectra of hVDAC1 in SDS indicated denatured hVDAC1 with high content of $\alpha$-helix secondary structure (- - ). CD spectra of hVDAC1 first incubated in LDAO $(-)$ and then reconstituted into SDS $(\cdots)$ were similar to CD spectra obtained by direct reaction with SDS. SDS bound tightly to hVDAC1 and could not be replaced by LDAO and dialysis $(-\cdot-\cdot)$. 
and a strong reduction in $\beta$-sheet structure in comparison to hVDAC1 in LDAO, suggesting a breakdown of the $\beta$-barrel structure of hVDAC1 in SDS. When hVDAC1 in SDS was reconstituted into LDAO micelles by dialysis, the previously observed secondary structure of hVDAC1 in LDAO could not be recovered, suggesting hVDAC1 denaturation in SDS and an irreversible tight binding of SDS to hVDAC1. The increased $\alpha$-helical structure of hVDAC1 in SDS is similar to observations for VDAC of N.crassa at $\mathrm{pH} 8 .^{12}$

\section{Discussion}

While the mechanism of membrane insertion and folding of bacterial membrane proteins into lipid bilayer membranes has been examined previously, we here investigated this process for the first time for a eukaryotic $\beta$-barrel membrane protein, the human VDAC isoform 1 of the OM of mitochondria. Our results revealed a new mechanism of membrane insertion and folding. First, VDAC developed a large content of $\beta$-sheet structure already in aqueous solution and, in addition to the $\beta$-sheet, $\alpha$-helical structure was formed upon folding of hVDAC1 in the presence of lipid bilayers. Second, hVDAC1 does not fold to its native form in detergent. Instead, a lipid bilayer is required for formation of correctly inserted, folded, and stable hVDAC1. Third, folding of hVDAC1 into bilayers of di $\mathrm{C}_{14: 0} \mathrm{PC}$, unlike OmpA, did not require small vesicles with high curvature stress, ${ }^{18}$ but was successful with large unilamellar vesicles, indicating a lower activation energy of the transition state for folding of hVDAC1. Similar to bacterial OMPs, folding and membrane insertion of hVDAC1 into lipid bilayers of phosphatidylcholines is spontaneous, and native structure formation in these bilayers does neither require proteinaceous folding machinery nor an energy source like ATP.

\section{hVDAC1 inserts and folds spontaneously into phospholipid bilayers}

At least two phases of structure formation could be distinguished upon folding of hVDAC1 into lipid bilayers (Figure 9). In a first step upon urea dilution, hVDAC1 quickly develops large amounts of $\beta$-sheet secondary structure in an aqueous intermediate $(\mathrm{A})$, while $\alpha$-helical structure was initially absent. In a second step of membrane insertion, about 25-30 residues $(8-10 \%)$ are converted from a random conformation to $\alpha$-helical structure, while $\beta$-sheet secondary structure formation is completed $(\mathrm{N})$. This natively folded form, $\mathrm{N}$, functioned as a voltage-

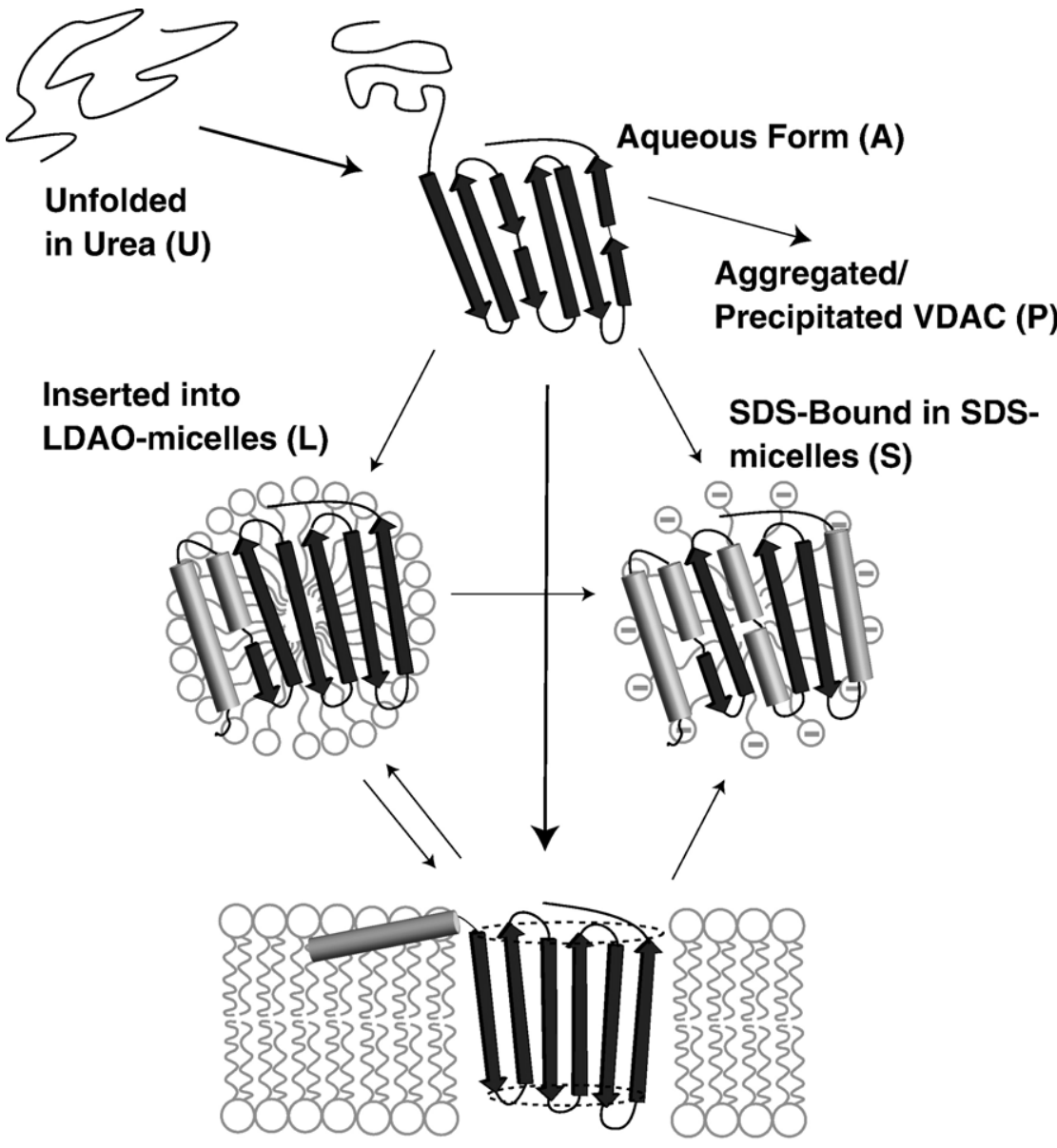

Native VDAC in Phospholipid Membranes (N)
Figure 9. A tentative scheme for folding and insertion of hVDAC1 into lipid bilayers. From an unfolded form in urea (U) that does not display helix or sheet content, after urea dilution, hVDAC1 develops $\sim 39 \% \quad \beta$-strand structure (110 residues) in an aqueous intermediate (A). From this intermediate, hVDAC1 folds and inserts directly into lipid bilayers to its native form $(\mathrm{N})$, composed of $\sim 11 \% \quad \alpha$-helix ( $\sim 30$ residues), $\sim 36 \% \quad \beta$-strand, and $23 \% \quad \beta$-turn structure. Upon incubation with LDAO, hVDAC1 develops $\sim 16 \% \quad \alpha$-helix (45 residues), $\sim 33 \% \quad \beta$-strand ( $\sim 93$ residues), and $23 \% \beta$-turn structure. Upon reconstitution into phospholipid bilayers this secondary structure is converted to the secondary structure observed for direct refolding of denatured hVDAC1 into phospholipid bilayers. In SDSmicelles, SDS binds tightly to hVDAC1 and leads to formation of $\sim 29 \% \alpha$-helical structure ( $\sim 82$ residues). The number of strands in these forms is not known. 
dependent channel with the same conductance states as VDACs that were previously isolated directly from membranes in native form without denaturation and refolding steps. ${ }^{9,20} \mathrm{~N}$ was stable over a wide $\mathrm{pH}$ range from 3 to 10 . The formation of $\alpha$-helical structure in the presence of bilayers is consistent with previous results on the amphipathic peptide composed of the $20 \mathrm{~N}$-terminal residues of VDAC. This peptide was unstructured in aqueous solution and developed $\alpha$-helical structure in non-polar solvent and SDS-micelles. ${ }^{7}$ From the CD spectra, it is obvious that the fraction of $\beta$-sheet secondary structure in aqueous hVDAC1 (Figure 1(a)) is much higher than in the aqueous intermediate of OmpA. ${ }^{17}$ The structure of the aqueous intermediate will affect the transition state of the membrane protein for insertion and native structure formation. While reduced vesicle size is critical for lowering the free energy of the transition state of OmpA folding, ${ }^{26}$ this is apparently not important for hVDAC1, which folds into LUVs of $d i C_{14: 0} \mathrm{PC}$. This is very likely a consequence of more $\beta$-sheet structure that we observed in the aqueous intermediate of hVDAC1 compared to previous observations for OmpA. ${ }^{17}$ In contrast to OmpA, folding and membrane insertion of hVDAC1 are at least in part uncoupled. Since there is more $\beta$-sheet structure in the aqueous folding intermediate of hVDAC1, subsequent transient and intermediate folding states of hVDAC1 will also contain higher amounts of $\beta$-strand secondary structure than those observed for OmpA of E. coli at a comparable stage of membrane insertion.

\section{Structure and stability of hVDAC1 in lipid bilayers and detergent micelles}

When the aqueous intermediate $A$ reacted with detergent micelles of LDAO, DDM, or $\mathrm{C}_{8} \mathrm{E}_{4}$, on average $\sim 45-54$ residues of hVDAC1 adopted $\alpha$-helical structure in the micelle solubilized form, L. Formation of $\alpha$-helix content ${ }^{6}$ required the presence of a lipid bilayer or a detergent micelle. While $\mathrm{L}$ could have a single unique structure at $\mathrm{pH}$ 3 , it was not thermodynamically stable at neutral $\mathrm{pH}$. L could be converted to $\mathrm{N}$ by adding it to lipid bilayers. $\mathrm{L}$ is likely not an intermediate in the direct folding-pathway, because this form can fold to the active form in diPhPC while direct folding into diPhPC from the urea-unfolded state was not possible in the absence of detergent.

The $\alpha$-helical content of L, was larger than in lipid bilayers by $\sim 15$ residues. Correspondingly, the $\beta$-strand fraction was smaller by 15 residues (Tables 1 and 2). Therefore, 1-2 TM $\beta$-strands may be additionally present in $\mathrm{N}$. This was consistent for all lipids, except for the thinnest bilayer, $d i \mathrm{C}_{10: 0} \mathrm{PC}$. $h$ VDAC1 did not form additional $\alpha$-helical structure in $\operatorname{diC}_{10: 0} \mathrm{PC}$, but an overall increase in $\beta$-turn and random structure at the expense of $\beta$-strand content was determined (Table 2). It is possible that the $\beta$ strands of hVDAC1 are not well matched by the hydrophobic thickness of $d \mathrm{iC}_{10: 0} \mathrm{PC}$ and destabilized at their ends at the membrane water interfaces.
Upon pepsinolysis of $\mathrm{L}$, an additional fragment of $\sim 24 \mathrm{kDa}$ can immediately be detected. Compared to the $26 \mathrm{kDa}$ fragment of hVDAC1 obtained in lipid bilayers, the cleavage of additional $\sim 20$ residues possibly corresponds to the segment that forms the additional $\alpha$-helical structure in detergents. Clearly, hVDAC1 forms a less stable three-dimensional fold than bacterial OMPs, since the secondary structure and resistance to proteolysis depend strongly on the environment of hVDAC1, whether it is a detergent micelle or a lipid bilayer. Proteolysis of bacterial OMPs in detergent micelles does neither result in multiple fragments nor is proteolysis complete within $1 \mathrm{~h} .{ }^{15}$ The reduced stability of hVDAC 1 in detergent is also evident by an increased $\mathrm{pH}$ sensitivity of the hVDAC1 secondary structure in micelles compared to bilayers (Figure 4(b)). Our observations suggest that hVDAC1 is present in partially folded/denatured form even in milder detergents than SDS. This could explain the difficulties in obtaining a high-resolution structure of a detergent solubilized VDAC. Mitochondrial OMPs may be less stable than bacterial OMPs, because mitochondria are not exposed to similar harsh environmental conditions as bacteria.

In SDS (S), hVDAC1 was irreversibly denatured and developed 29\% $\alpha$-helical structure at room temperature (RT) (Table 1). Folded OMPs of bacteria are usually stable in SDS at RT and migrate differently when not heat-denatured prior to SDSPAGE (see e.g. Kleinschmidt for a review ${ }^{3}$ ). This was not observed for hVDAC1 as a consequence of the altered secondary (and tertiary) structure in SDS. In detergent micelles, VDACs from other organisms might be more or less stable than hVDAC1. VDAC of N. crassa in LDAO at pH 8 contained $12 \% \alpha$-helix, while natively isolated VDAC of Saccharomyces cerevisiae contained $27 \% \alpha$-helix in LDAO at $\mathrm{pH} 8 .^{12}$

\section{Correct folding of $\mathrm{hVDAC} 1$ requires the lipid bilayer}

The composition of the secondary structure of hVDAC1 changed systematically with increased detergent content in mixtures of $\mathrm{LDAO} / \mathrm{diC}_{12: 0} \mathrm{PC}$, suggesting that the structure of the lipid bilayer is critical for the correct formation of hVDAC1 secondary structure and not just a few lipid molecules that may bind and stabilize the native fold. The lower the amount of LDAO present the more native is the structure of hVDAC1. At a mole fraction of LDAO of 0.5 , the fractions of $\alpha$-helical and $\beta$-sheet structure in hVDAC 1 are close to the average of those at 0 and at $100 \%$ LDAO. Thus either half of hVDAC1 has a structure typical for hVDAC1 in lipid bilayers and the other half a structure typical for those in detergent micelles or all of hVDAC1 has some intermediate structure. To clarify this question, a phase diagram for $\mathrm{LDAO} / \mathrm{diC}_{12} \mathrm{PC}$ mixtures would be needed but has not yet been reported and it will be interesting to investigate this question in a future study.

The formation of different structures of hVDAC1 in micelles and in bilayers distinguishes hVDAC1 
strongly from the previously investigated folding behavior of prokaryotic OMPs, which have the same secondary structure in lipid bilayers and in detergent micelles as shown for FomA ${ }^{15}$ and OmpA ${ }^{27}$ and which do not fold into bilayers of large unilamellar vesicles of $d i \mathrm{C}_{14: 0} \mathrm{PC}$, but require their sonication.

\section{Implications of spontaneous folding of hVDAC1 in lipid bilayers for assembly in vivo}

hVDAC1 folds spontaneously into lipid bilayers of phosphatidylcholine, similar to the bacterial outer membrane proteins OmpA and FomA. While phosphatidylcholine is a major lipid component $(\sim 50 \%)$ of the mitochondrial outer membrane, the physical properties of the mitochondrial outer membrane are possibly different to prevent spontaneous insertion and to provide a selection mechanism for specific membrane proteins. For this reason, proteinaceous machinery may be required for membrane insertion of mitochondrial ${ }^{28-30}$ and bacterial ${ }^{31-33}$ outer membrane proteins in vivo. Even while this may be the case, our biophysical work provides new insight to the in vivo assembly process. Membrane insertion and folding of $\beta$-barrel membrane proteins of the mitochondrial outer membrane, in contrast to the in vivo assembly process of $\alpha$-helix transmembrane proteins, neither absolutely requires proteinaceous machinery nor energy sources such as ATP to obtain the native fold of hVDAC1 upon membrane insertion. Our observations instead point toward the need of membrane embedded or membrane-associated folding assistants for other reasons than energy-driven translocation. These reasons may be: (1) to provide a specific targeting mechanism for mitochondrial outer membrane proteins, (2) to displace chaperones needed to maintain outer membrane proteins soluble in the inter membrane space and possibly (3) to alter the physical properties of the membrane in their direct vicinity for transmembrane protein insertion.

The observations of spontaneous and oriented folding, first for bacterial OMPs like OmpA and FomA and now for mitochondrial hVDAC1 emphasize the energy efficiency of the cell, which has been conserved during evolution. In contrast to the periplasm of bacteria, there is ATP in the inter membrane space of mitochondria. Although this energy source is present, folding and insertion of mitochondrial hVDAC1 can, by physical principle, occur spontaneously similar to folding of bacterial OMPs. The gain in free energy by formation of the native fold of hVDAC1 in the mitochondrial outer membrane and of bacterial OMPs in outer membranes is sufficient to cause their folding and membrane insertion.

From a physical point of view, the requirement of lipid bilayers for formation of native and stable structure in hVDAC1, the spontaneous insertion into bilayers of LUVs (versus SUVs), and the formation of both $\beta$-barrel and $\alpha$-helical structure, indicated a fundamentally different sequence of structure formation in mitochondrial hVDAC1 and bacterial OMPs, for which folding has been investigated to date. It will be interesting to compare the sequence of folding events with bacterial membrane proteins that also contain an $\alpha$-helical terminus, for example PagP. $^{34}$

\section{Materials and Methods}

\section{Expression and purification of hVDAC1}

The hvdac1 (REFSEQ(NM): NM_003374) PCR product (849 bp) and the pET22b(+) (Novagen, $5493 \mathrm{bp}$ ) expression vector were digested with NdeI and XhoI restriction endonuleases and ligated. The resulting plasmid pTMVDAC1 contained the gene for hVDAC1 expression under control of the T7 promoter and the gene for ampicillin resistance for plasmid selection. pTMVDAC1 was transformed into E.coli strain BL21(DE3)omp8 fhuA $\left[\mathrm{F}^{-}\right.$,ompT $h s d S_{\mathrm{B}}\left(\mathrm{r}_{\mathrm{B}}^{-} \mathrm{m}_{\mathrm{B}}^{-}\right) \mathrm{gal} d c m$ (DE3) $\Delta \operatorname{lamB}$ ompF::Tn5 $\Delta$ OmpA $\Delta$ OmpC $\Delta$ fhuA ${ }^{35}$ using the TSS method. ${ }^{36}$

Cells were grown at $37^{\circ} \mathrm{C}$ for $\sim 3 \mathrm{~h}$ in LB medium (containing $0.1 \mathrm{~g} / 1 \mathrm{ampicillin}$ and $0.05 \mathrm{~g} / 1$ neomycin) to $A_{600 \mathrm{~nm}}=0.9$. After $\sim 5-6 \mathrm{~h}$ of induction with $1 \mathrm{mM}$ isopropyl- $\beta$-D-thiogalactopyranoside, cells were centrifuged at $5000 \mathrm{rpm}$ for $30 \mathrm{~min}$, resuspended in buffer A (25 mM Tris (pH 8.0), $150 \mathrm{mM} \mathrm{NaCl}, 0.2 \mathrm{mM}$ PMSF) and broken at 2.69 kbar using a cell disruption system (Constant Systems Ltd., Daventry, England). Inclusion bodies (IBs) of hVDAC1 were obtained by $30 \mathrm{~min}$ of ultracentrifugation at $51,500 \mathrm{~g}$ at $4{ }^{\circ} \mathrm{C}$. IBs were homogenized with buffer $\mathrm{A}$, centrifuged, washed with buffer (25 mM Tris ( $\mathrm{pH} 8.0), 150 \mathrm{mM} \mathrm{NaCl}, 1 \%$ Triton X-100). The suspension was washed with buffer $\mathrm{A}$ and centrifuged at $33,000 \mathrm{~g}$ for $30 \mathrm{~min}$. IBs of hVDAC1 were resuspended in $100 \mathrm{mM}$ Tris ( $\mathrm{pH} 8.0$ ), $10 \mathrm{mM} \mathrm{1,4-}$ dithiothreitol, $1 \mathrm{mM}$ EDTA containing $8 \mathrm{M}$ urea, solubilized overnight at $10{ }^{\circ} \mathrm{C}$, and then purified over a DEAE Sepharose column (Amersham Biosciences, Sweden) in $50 \mathrm{mM}$ Tris ( $\mathrm{pH} 7.5)$, containing $8 \mathrm{M}$ urea. hVDAC1 was then dialyzed overnight against $50 \mathrm{mM}$ Mes ( $\mathrm{pH}$ 6), $8 \mathrm{M}$ urea loaded onto a CM Sepharose column (Amersham) and eluted by a linear gradient of 0 $500 \mathrm{mM} \mathrm{NaCl}$. Fractions were pooled, concentrated, and hVDAC1 concentration was determined. ${ }^{24}$

\section{Preparation of lipid bilayers}

Phospholipids (Avanti Polar Lipids, Alabaster, AL) were dissolved in chloroform, dried under a stream of nitrogen and in a desiccator under high vacuum to prepare thin lipid films. Lipid films were hydrated in $10 \mathrm{mM}$ citrate buffer ( $\mathrm{pH}$ 3.0, with $2 \mathrm{mM}$ EDTA) and dispersed by vortexing. LUVs were prepared by seven cycles of freeze-thawing the hydrated lipids in liquid nitrogen and in a water bath at $35{ }^{\circ} \mathrm{C}$. Lipid dispersions were then extruded 30 times through $100 \mathrm{~nm}$ pore size polycarbonate membranes (Nucleopore, Whatman, Clifton, NJ) using a mini-extruder (Avanti, Alabaster, AL). LUVs were used in folding experiments on the same day.

\section{Folding of hVDAC1 into detergent micelles or lipid bilayers}

Folding of hVDAC1 was initiated by 40 -fold dilution of hVDAC1 into citrate buffer containing an 800-fold molar excess of phospholipids, LDAO (Fluka, Buchs, Switzerland), DDM, or $\mathrm{C}_{8} \mathrm{E}_{4}$, or a 640 -fold molar excess of SDS, at 
a final hVDAC1 concentration of $16.3 \mu \mathrm{M}$. All samples were incubated $5 \mathrm{~h}$ at $25{ }^{\circ} \mathrm{C}$. To remove residual urea, samples were dialyzed overnight against 11 of citrate buffer with three buffer replacements. At alternative $\mathrm{pH}$, Tris $(\mathrm{pH} 8)$, sodium tetraborate $(\mathrm{pH} \mathrm{10})$ and Caps $(\mathrm{pH} 11)$ were used as buffers. Reconstitutions from detergent into lipid were performed at $\mathrm{pH} 3$. For samples at $\mathrm{pH} 7$, we added 1.2 volumes of $50 \mathrm{mM}$ Hepes buffer $(\mathrm{pH} 7)$.

\section{CD spectroscopy}

Far UV CD spectra were recorded at RT by a Jasco 715 CD spectrometer (Jasco, Tokyo, Japan) using a $0.5 \mathrm{~mm}$ cuvette. Three scans were accumulated from 190 to $250 \mathrm{~nm}$ with a response time of $8 \mathrm{~s}$, a bandwidth of $1 \mathrm{~nm}$ and a scan speed of $20 \mathrm{~nm} / \mathrm{min}$. Background spectra without hVDAC1 were subtracted. The concentrations of hVDAC1 were determined for each sample. ${ }^{24}$ The recorded CD spectra were normalized to the mean residue molar ellipticity ${ }^{37}(\lambda)$, given by:

$$
[\Theta](\lambda)=100 \frac{\Theta(\lambda)}{c \cdot n \cdot l},
$$

where $l$ is the path length of the cuvette in $\mathrm{cm}, \Theta(\lambda)$ is the recorded ellipticity in degrees at wavelength $\lambda, c$ is the concentration in mol/l, and $n$ the number of amino acid residues of hVDAC1 (282). Spectra were analyzed using DICHROWEB (see Whitmore and Wallace ${ }^{38}$ and references therein). Several deconvolution programs (CONTIN, ${ }^{39}$ SELCON3 ${ }^{40}$ and CDSSTR $^{41}$ ) were used for analysis.

\section{Fluorescence spectroscopy}

Fluorescence spectra of hVDAC1 $(1.3 \mu \mathrm{M})$ were recorded at $25{ }^{\circ} \mathrm{C}$ on a Spex Fluorolog-3-22 fluorometer. Background spectra were subtracted. The excitation wavelength was $295 \mathrm{~nm}$. The bandwidths of the excitation and emission monochromators were $2 \mathrm{~nm}$ and $5 \mathrm{~nm}$, respectively. The integration time was $0.05 \mathrm{~s}$ and an increment of $0.5 \mathrm{~nm}$ was used to scan spectra in the range of 305-400 nm. Three scans were averaged for each spectrum.

\section{Proteolysis}

$50 \mu \mathrm{l}$ hVDAC1 $(0.5 \mathrm{~g} / \mathrm{l})$ were incubated with $2.5 \mu \mathrm{l}$ pepsin (Fluka) $\left(0.1 \mathrm{~g} / 1\right.$ in $2 \%$ acetic acid) at $37{ }^{\circ} \mathrm{C}$ under gentle shaking for at least $30 \mathrm{~min}$. Proteolysis was stopped by adding $1 \mu \mathrm{l}$ of $1 \mathrm{M} \mathrm{NaOH}$. Samples were analyzed by SDS-PAGE.

\section{Single-channel conductance across black lipid bilayers}

The single channel conductance across diPhPC bilayers was recorded as described, ${ }^{15}$ but with Tris buffer $(10 \mathrm{mM}$ Tris ( $\mathrm{pH} 7.4$ ), $5 \mathrm{mM} \mathrm{CaCl}_{2}$ containing $1 \mathrm{M} \mathrm{KCl}$ ) in the twocompartment Teflon cell. 0.05 to $0.3 \mu \mathrm{g} / \mathrm{ml}$ of hVDAC1 in LDAO was added to the front compartment. A potential of $10 \mathrm{mV}$ was applied to the front electrode. To determine the voltage-dependence of the single-channel conductance, hVDAC1 was added to both sides of a black diPhPC membrane to a final concentration of $250 \mathrm{ng} / \mathrm{ml}$. After $10 \mathrm{~min}$, an initial potential of $10 \mathrm{mV}$ was applied and the conductance $G_{0}$ was calculated from the recorded current. The potential was then increased in increments of $+10 \mathrm{mV}$, up to $+60 \mathrm{mV}$ and the relative conductance $\left(G / G_{0}\right)$ of
hVDAC1 was obtained. Similarly, the relative conductance was obtained for negative potentials. The normalized conductance $\left(G / G_{0}\right)$ was plotted as a function of the applied voltage.

\section{Sucrose density gradient centrifugation}

Linear gradients from 5 to $30 \%(\mathrm{w} / \mathrm{v})$ sucrose were prepared in a $25 \mathrm{ml}$ centrifuge tube using a gradient mixer (University of Konstanz). $0.3 \mathrm{mg}$ of folded hVDAC1 in $d \mathrm{C}_{12: 0} \mathrm{PC}$ in citrate buffer were layered on the top of the gradient and centrifuged at $132,000 \mathrm{~g}$ at $4{ }^{\circ} \mathrm{C}$ for $4 \mathrm{~h}$. After centrifugation, the two obtained layers were collected separately with a Pasteur pipette and their protein and lipid contents were estimated as described. ${ }^{24,42}$

\section{Acknowledgements}

This work was supported by grants KL 1024/4-1 and 2 from the Deutsche Forschungsgemeinschaft to J.H.K. and, for construction of the pTMVDAC1 plasmid, by DFG grant ZE522/1-1 to K.Z.

\section{References}

1. Engelman, D. M., Chen, Y., Chin, C. N., Curran, A. R., Dixon, A. M., Dupuy, A. D. et al. (2003). Membrane protein folding: beyond the two stage model. FEBS Letters, 555, 122-125.

2. Booth, P. J. (2005). A paradigm of membrane protein folding: principles, kinetics and stability of bacteriorhodopsin folding. In Protein-Lipid Interactions: From Membrane Domains to Cellular Networks (Tamm, L. K., ed), pp. 57-80, Wiley-VCH, Weinheim.

3. Kleinschmidt, J. H. (2006). Folding kinetics of the outer membrane proteins OmpA and FomA into phospholipid bilayers. Chem. Phys. Lipids, 141, 30-47.

4. Colombini, M. (2004). VDAC: the channel at the interface between mitochondria and the cytosol. Mol. Cell. Biochem. 256-257, 107-115.

5. Wiedemann, N., Truscott, K. N., Pfannschmidt, S., Guiard, B., Meisinger, C. \& Pfanner, N. (2004). Biogenesis of the protein import channel Tom40 of the mitochondrial outer membrane: intermembrane space components are involved in an early stage of the assembly pathway. J. Biol. Chem. 279, 18188-18194.

6. Song, J., Midson, C., Blachly-Dyson, E., Forte, M. \& Colombini, M. (1998). The topology of VDAC as probed by biotin modification. J. Biol. Chem. 273, 24406-24413

7. Guo, X. W., Smith, P. R., Cognon, B., D'Arcangelis, D., Dolginova, E. \& Mannella, C. A. (1995). Molecular design of the voltage-dependent, anion-selective channel in the mitochondrial outer membrane. J. Struct. Biol. 114, 41-59.

8. Abu-Hamad, S., Sivan, S. \& Shoshan-Barmatz, V. (2006). The expression level of the voltage-dependent anion channel controls life and death of the cell. Proc. Natl Acad. Sci. USA, 103, 5787-5792.

9. Benz, R. (1994). Permeation of hydrophilic solutes through mitochondrial outer membranes: review on mitochondrial porins. Biochim. Biophys. Acta, 1197, 167-196.

10. Abrecht, H., Goormaghtigh, E., Ruysschaert, J. M. \& Homble, F. (2000). Structure and orientation of two voltage-dependent anion-selective channel isoforms. 
An attenuated total reflection fourier-transform infrared spectroscopy study. J. Biol. Chem. 275, 40992-40999.

11. Casadio, R., Jacoboni, I., Messina, A. \& De Pinto, V. (2002). A 3D model of the voltage-dependent anion channel (VDAC). FEBS Letters, 520, 1-7.

12. Koppel, D. A., Kinnally, K. W., Masters, P., Forte, M. Blachly-Dyson, E. \& Mannella, C. A. (1998). Bacterial expression and characterization of the mitochondrial outer membrane channel. Effects of N-terminal modifications. J. Biol. Chem. 273, 13794-13800.

13. Shao, L., Kinnally, K. W. \& Mannella, C. A. (1996). Circular dichroism studies of the mitochondrial channel VDAC, from Neurospora crassa. Biophys. J. 71, 778-786.

14. Cowan, S. W., Garavito, R. M., Jansonius, J. N. Jenkins, J. A., Karlsson, R., Konig, N. et al. (1995). The structure of OmpF porin in a tetragonal crystal form. Structure, 3, 1041-1050.

15. Pocanschi, C. L., Apell, H.-J., Puntervoll, P., Høgh, B T., Jensen, H. B., Welte, W. \& Kleinschmidt, J. (2006). The major outer membrane protein of Fusobacterium nucleatum (FomA) folds and inserts into lipid bilayers via parallel folding pathways. J. Mol. Biol. 355, 548-561.

16. Buchanan, S. K. (1999). $\beta$-Barrel proteins from bacterial outer membranes: structure, function and refolding. Curr. Opin. Struct. Biol. 9, 455-461.

17. Kleinschmidt, J. H. \& Tamm, L. K. (2002). Secondary and tertiary structure formation of the $\beta$-barrel membrane protein OmpA is synchronized and depends on membrane thickness. J. Mol. Biol. 324, 319-330.

18. Surrey, T. \& Jähnig, F. (1992). Refolding and oriented insertion of a membrane protein into a lipid bilayer. Proc. Natl Acad. Sci. USA, 89, 7457-7461.

19. Surrey, T., Schmid, A. \& Jähnig, F. (1996). Folding and membrane insertion of the trimeric $\beta$-barrel protein OmpF. Biochemistry, 35, 2283-2288.

20. Blachly-Dyson, E., Zambronicz, E. B., Yu, W. H., Adams, V., McCabe, E. R., Adelman, J. et al. (1993). Cloning and functional expression in yeast of two human isoforms of the outer mitochondrial membrane channel, the voltage-dependent anion channel. J. Biol. Chem. 268, 1835-1841.

21. Benz, R. (1990). Biophysical properties of porin pores from mitochondrial outer membrane of eukaryotic cells. Experientia, 46, 131-137.

22. Colombini, M. (1989). Voltage gating in the mitochondrial channel, VDAC. J. Membr. Biol. 111, 103-111.

23. Liu, M. Y. \& Colombini, M. (1992). Regulation of mitochondrial respiration by controlling the permeability of the outer membrane through the mitochondrial channel, VDAC. Biochim. Biophys. Acta, 1098, 255-260.

24. Lowry, O. H., Rosebrough, N. J., Farr, A. L. \& Randall R. J. (1951). Protein measurement with the Folin phenol reagent. J. Biol. Chem. 193, 265-275.

25. Bollag, D. M. \& Edelstein, S. J. (1991). Protein Methods, Wiley-Liss, New York.

26. Pocanschi, C. L., Patel, G. J., Marsh, D. \& Kleinschmidt, J. H. (2006). Curvature elasticity and refolding of OmpA in large unilamellar vesicles. Biophys. J. 91, L75-L78.

27. Kleinschmidt, J. H., Wiener, M. C. \& Tamm, L. K. (1999). Outer membrane protein A of E. coli folds into detergent micelles, but not in the presence of monomeric detergent. Protein Sci. 8, 2065-2071.
28. Ishikawa, D., Yamamoto, H., Tamura, Y., Moritoh, K. \& Endo, T. (2004). Two novel proteins in the mitochondrial outer membrane mediate $\beta$-barrel protein assembly. J. Cell Biol. 166, 621-627.

29. Kozjak, V., Wiedemann, N., Milenkovic, D., Lohaus, C., Meyer, H. E., Guiard, B. et al. (2003). An essential role of Sam50 in the protein sorting and assembly machinery of the mitochondrial outer membrane. J. Biol. Chem. 278, 48520-48523.

30. Paschen, S. A., Waizenegger, T., Stan, T., Preuss, M., Cyrklaff, M., Hell, K. et al. (2003). Evolutionary conservation of biogenesis of $\beta$-barrel membrane proteins. Nature, 426, 862-866.

31. Voulhoux, R., Bos, M. P., Geurtsen, J., Mols, M. \& Tommassen, J. (2003). Role of a highly conserved bacterial protein in outer membrane protein assembly. Science, 299, 262-265.

32. Werner, J. \& Misra, R. (2005). YaeT (Omp85) affects the assembly of lipid-dependent and lipid-independent outer membrane proteins of Escherichia coli. Mol. Microbiol. 57, 1450-1459.

33. Wu, T., Malinverni, J., Ruiz, N., Kim, S., Silhavy, T. J. \& Kahne, D. (2005). Identification of a multicomponent complex required for outer membrane biogenesis in Escherichia coli. Cell, 121, 235-245.

34. Hwang, P. M., Choy, W. Y., Lo, E. I., Chen, L., FormanKay, J. D., Raetz, C. R. et al. (2002). Solution structure and dynamics of the outer membrane enzyme PagP by NMR. Proc. Natl Acad. Sci. USA, 99, 13560-13565.

35. Prilipov, A., Phale, P. S., Van Gelder, P., Rosenbusch, J. P. \& Koebnik, R. (1998). Coupling site-directed mutagenesis with high-level expression: large scale production of mutant porins from E. coli. FEMS Microbiol. Letters, 163, 65-72.

36. Ausubel, F. M., Brent, R., Kingston, R. E., Moore, D., Seidman, J. G., Smith, J. A. \& Struhl, K. (eds.). (1999). Short Protocols in Molecular Biology, 4th edit. John Wiley and Sons, New York.

37. Abrams, F. S. \& London, E. (1992). Calibration of the parallax fluorescence quenching method for determination of membrane penetration depth: refinement and comparison of quenching by spin-labeled and brominated lipids. Biochemistry, 31, 5312-5322.

38. Whitmore, L. \& Wallace, B. A. (2004). DICHROWEB, an online server for protein secondary structure analyses from circular dichroism spectroscopic data. Nucl. Acids Res. 32, W668-W673.

39. Provencher, S. W. \& Glockner, J. (1981). Estimation of globular protein secondary structure from circular dichroism. Biochemistry, 20, 33-37.

40. Sreerama, N., Venyaminov, S. Y. \& Woody, R. W. (1999). Estimation of the number of $\alpha$-helical and betastrand segments in proteins using circular dichroism spectroscopy. Protein Sci. 8, 370-380.

41. Compton, L. A. \& Johnson, W. C., Jr (1986). Analysis of protein circular dichroism spectra for secondary structure using a simple matrix multiplication. Anal. Biochem. 155, 155-167.

42. Rouser, G., Fleischer, S. \& Yamamoto, A. (1970). Two dimensional then layer chromatographic separation of polar lipids and determination of phospholipids by phosphorus analysis of spots. Lipids, 5, 494-496. 